\title{
WaypostEye - a Software Tool for Navigation Improvement Support of Adaptive Websites
}

\author{
Željko Eremić $^{1}$, Dragica Radosav ${ }^{2}$ \\ ${ }^{1}$ Technical College of Applied Sciences, Djordja Stratimirovića bb, 23000 \\ Zrenjanin, Serbia, zeljko.eremic@vts-zr.edu.rs \\ ${ }^{2}$ University of Novi Sad, Technical faculty "Mihajlo Pupin", Djure Djakovića bb, \\ 23000 Zrenjanin, Serbia, radosav@tfzr.uns.ac.rs
}

\begin{abstract}
One frequently used method for navigation improvement of web sites is to establish a link between resources such as web pages or files, when it is fully justified. Adaptive web sites may change their display and structure based on a previously recorded user's behavior. The software tool that is described in this paper provides the designer with a graphical presentation of established link proposals between resources. In this way, it supports the designer's decisions referring to establishing new links between resources. Therefore, it makes a contribution to the improvement of the navigational structure of such web sites. This software establishes decision support information and Web designers are able to make business decisions in a relatively easy and visual way.
\end{abstract}

Keywords: adaptive website; waypost; website design

\section{Introduction}

When designing a website, an important aspect is to check the resource connections (web pages, files) within a site. The navigation structure in the web site is often not in keeping with the wishes and expectations of visitors. It often appears that a user must visit a number of web pages in order to get to the desired content. Well placed links on the pages can make a significant contribution to faster and easier access to the desired content. "Providing a link (i.e., shortcut) between these potential wayposts could assist users by reducing the number of clicks they have to make while browsing, pointing them in the right direction towards a specific target document." [1].

Adaptive web sites can customize their look and structure to meet the aspirations of user for fast and comfortable navigation. Users' behavior (navigation), previously recorded in log files, is used for the detection of the users' objective. By the appropriate processing of these data, it is possible to obtain the estimates of 
the resources that need to be connected using the hyperlinks. Each proposed hyperlink is evaluated on the basis of contributions to the navigation improvement. Based on the list of suggested links, the connections can be made between the resources, either automatically or manually added by the designer.

If the resources, which are located in the existing navigation, can be connected by adding links, these links can significantly accelerate the navigation through the website. This paper aims to present a software tool called WaypostEye which can graphically represent the distances between directly related web pages. In this way, the decision support is provided in the re-designing process of adaptive website navigation. Although the software tool is ready for use, its main function is to present the ideas on which it is based.

\section{Previous Studies}

Adaptive web sites are defined in [12] as "web sites that improve themselves by learning from user access patterns". In the previously mentioned article it is described the improvement in webmaster' understanding the interaction among visitors and the web site. Adaptive web sites often use information about previous user's behavior which is stored in log files. That information is in the standard form which is suitable for computer processing. General details referring to the $\log$ files are given in [11].

Shortcutting is a common way to improve the navigation on the web site. The article [1] considers using waypost documents as a relevant factor in process of improving classic shortcutting. Two illustrations of identifying waypost documents are given in [8].

Interaction events could be recorded for various digital objects (e.g. web pages). Those recordings could be used for better understanding of digital objects. Article [9] introduces history-enriched digital objects, describes a few prototypes and discusses privacy issues. The limited set of information stored in log files could be combined with the use of history-enriched digital objects, which has no such limitation. One approach comes from The Footprints project [14], which allows users to create history-rich digital objects in digital environment. Framework and software tools for visualization of navigational structure are developed to support the theory which claims that activities of past users could help new users in navigation through the space of information.

Displaying web site's navigational structure, for its improvement purposes, can be a challenging task. Fisheye technique implemented in hyperbolic browser, presented in [10], can visualize large hierarchies. The method for getting list of proposed shortcuts, based on recordings in log files, is explained in details within [5]. Its output is one of inputs in WaypostEye software tool. 
There are many areas where improved web site's navigation can bring benefits, and one of them is education. One approach to reduce omitting relevant documents and avoid user's withdraw from the web site is given in [6]. This approach reduces the number of clicks, avoiding visits to non-interesting documents (web pages) by offering links to potentially interesting documents.

While log files enable a better navigation from past information, there are possibilities to get help in navigation at the moment of browsing. One possibility is given in [7], where one user can ask another for help in a navigation process. That possibility is the combination of adaptive web site and Ajax. Historyenriched digital objects in combination with Ajax and database could be used for storing information about the knowledge sharing among users in real time, as described in [4]. Those objects in combination with log files could be great help for designing the efficient navigation of adaptive web site. Article [4] gives a conceptual and physical model, for the database required for recording knowledge in real time.

A parallel could be drawn with an interesting idea of Flexible Graphical User Interface presented in [2]. Proposed intuitive interface significantly reduces the task execution time and the number of interactions in the communication between a man and a machine.

\section{WaypostEye}

WaypostEye is a software tool that, graphically and appropriately, presents the optimal navigation proposal to the website designer. It uses the data from the input files and the results can be displayed in a number of ways.

The software tool (Figure 1) that is presented in this paper uses two files as the input where each file contains the correct type of information in the prescribed format:

- Structure File - Navigational structure file which contains the information about the existing relationships between resources (web pages or files). The first element of the pair is a resource which has a hyperlink to the second element of the pair.

- Shortcuts File - The file that contains the proposed links between documents based on the previous analyses of user's behavior. The first element of the pair is a resource with a proposed hyperlink to the second element of the pair. 


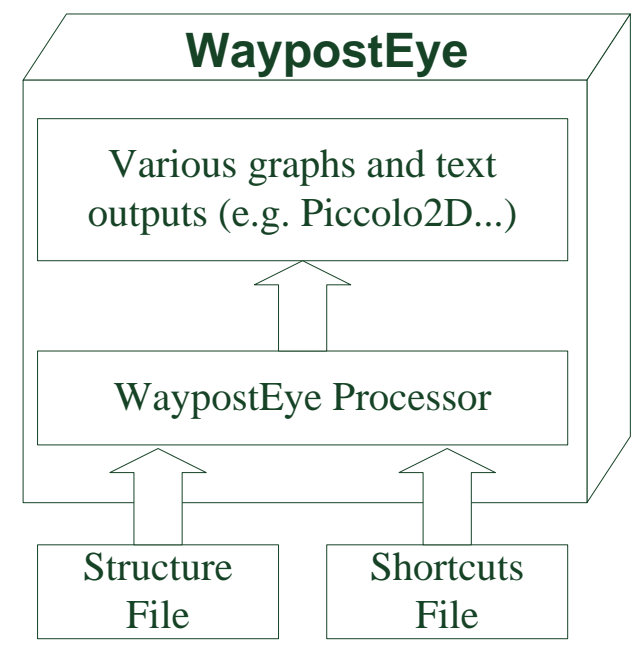

Figure 1

Input files and components of WaypostEye tool

"WaypostEye Processor" is a part of WaypostEye tool which stands between the input data (files) and the particular display of processing results. This part accepts the input data and provides a list of resources possible to perform the analysis of the current ones and recommended connections to other resources. When the user selects the appropriate resource, the graphical illustration of existing connections of this resource to other resources is given, as well as a proposal for the establishment of new hyperlinks.

The results can be graphically and textually presented. In this paper the results are presented using a graph based on a free and open source solution [13], but the list of possible outputs can be easily expanded.

The tool will be presented using the example of a simple web site which contains numerous resources. Each of the resources, as it is shown in the example, is associated with one or more resources, but there is no resource that is connected to all other resources. For the sake of simplicity, the simple rule has been established in this example - if resource A has a link to resource B, then resource B has the link to the resource A. Furthermore, four proposals for establishing connections between the unrelated pairs of resources have been also given. The navigation structure of the observed example and connection proposals are given in Figure 2. 


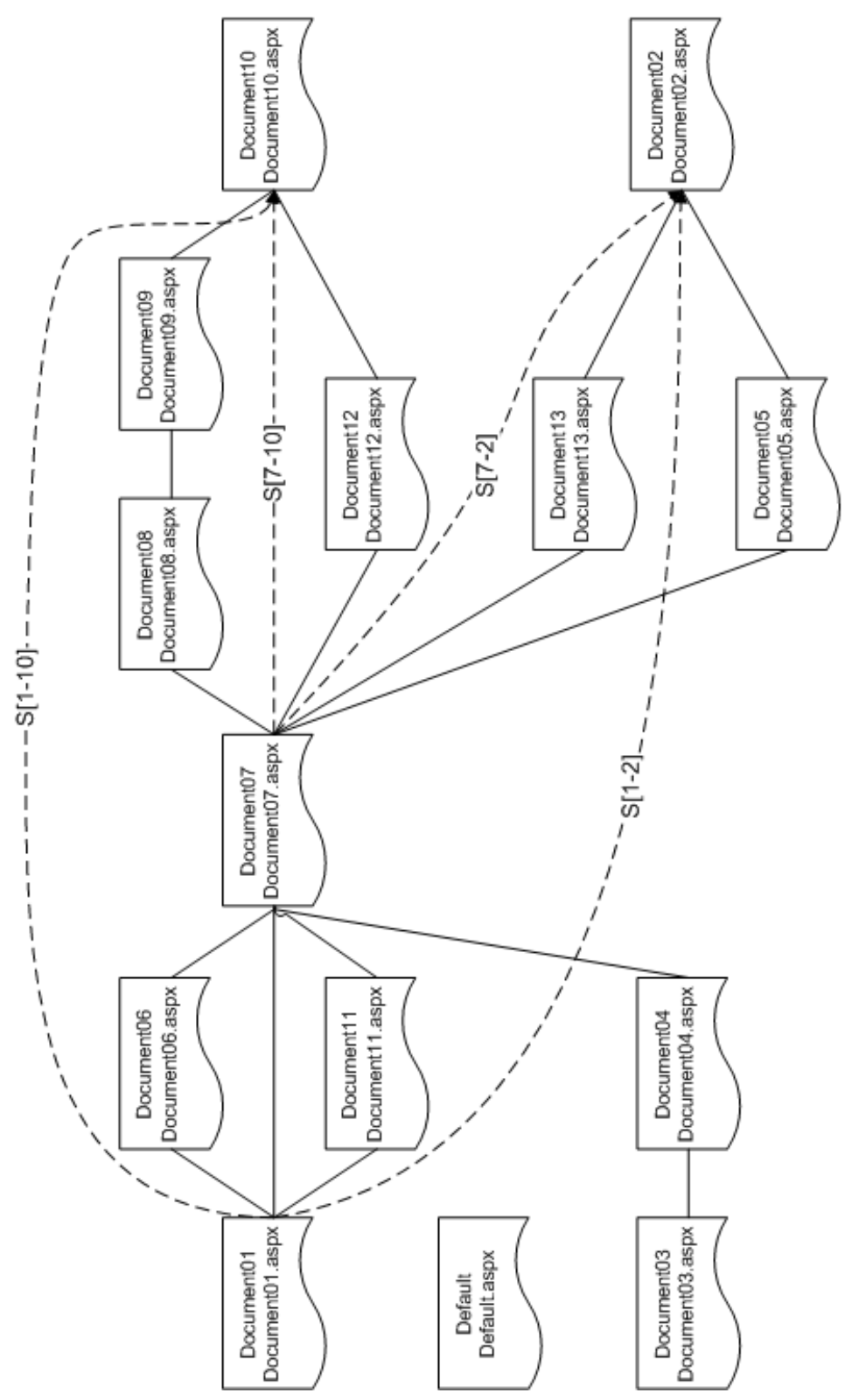

Figure 2

The example of a navigational structure, the connections are represented by solid lines, and suggested links (shortcuts) in dotted lines. Resources are represented by the title and the name of its file. 


\section{Input Data}

The first file (the navigation structure file) contains two kinds of information related to the existing navigation structure:

- Arranged pairs of resources that, are currently directly related.

- Title - Path pairs which connect the resource name with the path to the resource.

A console application which requests entering a web location has been created for the purpose of this research work (in the example, the experimental web site is located at localhost:54954/site43/). The application also requests entering the name of the file in which the analysis results of the existing navigation structure will be put. Figure 3 shows an example of console applications, and Figure 4 shows the parts of the file "structure.xml" containing the analysis results of console applications. The same result could be obtained by the expression in the command line "Crawler.exe localhost:54954/site43/ structure".

The previously mentioned $\mathrm{xml}$ file contains, in its first part, the arranged pairs of resources. By analyzing the HTML code, it was clear that there was a connection between those pairs of resources. The resource with a tag Source contains a link to a resource with a tag Destination. It should be emphasized that, if there is a connection from resource $\mathrm{A}$ to resource $\mathrm{B}$, it does not necessarily mean that a link from $\mathrm{B}$ to the resource $\mathrm{A}$ exists. The connections between the name of the resource and its path are in the second part of the file.

The second file contains output data (in XML format) from the software described in [5]. This file, which example is shown in Figure 5, contains pairs of resources for which establishing connections is recommended.

\section{Experiment}

The experiment demanded adequate software support. The development environment used was Visual Studio 2008. The test site with the appropriate web pages was made with ASP.NET technology. Web pages were given appropriate headings (Title attribute), and they were associated with other Web pages in accordance with Figure 2. By using the $\mathrm{C} \#$ programming language, a console application "Crawler" was made. It was used to analyze the structure of the existing website and store the results in an XML file. The software called "WaypostEye" was also made using C\#. 


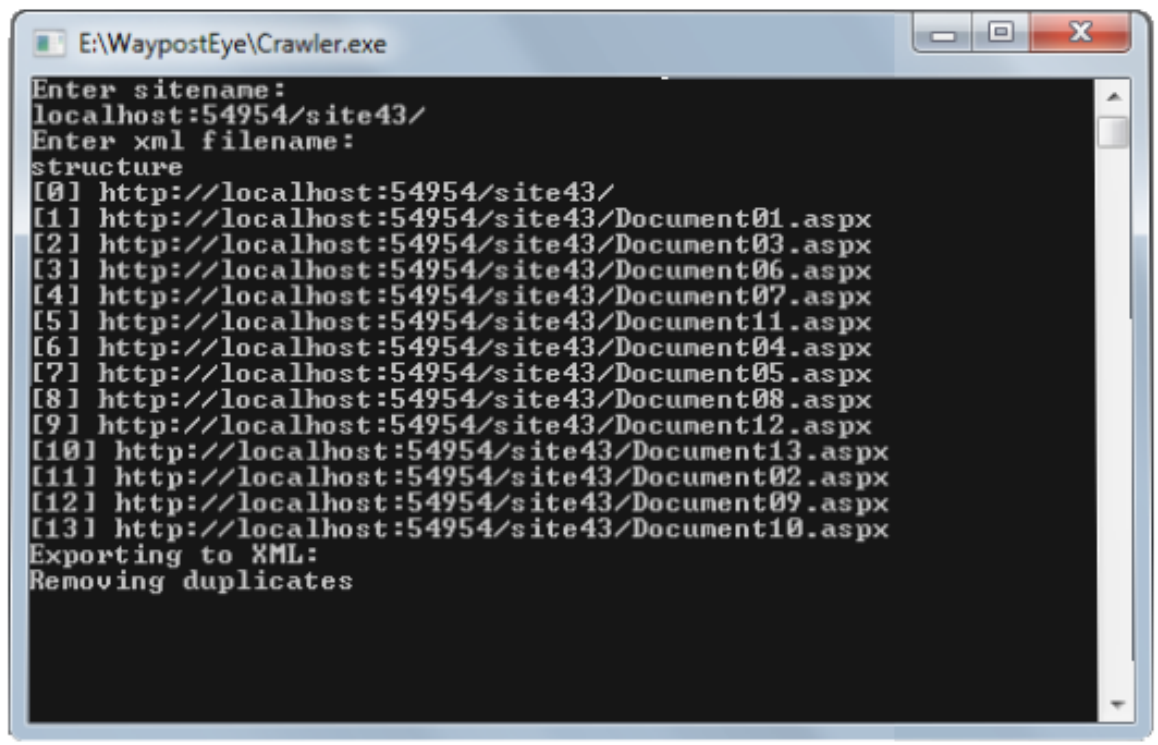

Figure 3

The console application that performs analysis of the site (localhost:54954/site43/) and stores the analysis results in the file (structure. $\mathrm{xml}$ )

Running the software takes us to the main window (Figure 6). The previously mentioned two input files need to be loaded. Afterwards, the list of resources of observed site is obtained in the lower part of the window. Appropriate transformations are done in WaypostEye software and finally, the output data are obtained. These data can be presented in different textual and graphical ways. Only graphical output based on the use of Piccolo2D [13], is supported at this stage of software development (version 1.0).

At this point, a resource needs to be selected from the lower section, in order to obtain the display of the existing connections for the resource, as well as, the connections of resources which should be associated with the observed document based on the content of the shortcut file. The result is the graph, in Figure 7. The documents are presented by their names inscribed in the rectangles of different colors. This document is inscribed into a black rectangle. The documents, associated with this selected document, are presented by a light gray rectangle and they are circularly arranged in relation to this document.

The paths towards the documents, which should be connected to the current document, are also presented on the graph. If there are multiple paths between documents, the shortest one is selected using Dijkstra's algorithm, described in [3]. In the given example, the selected "Document-7" should be connected to the "Document-2" (currently connected via the "Document-5"), and "Document-10" (currently connected via the "Document-12"). 


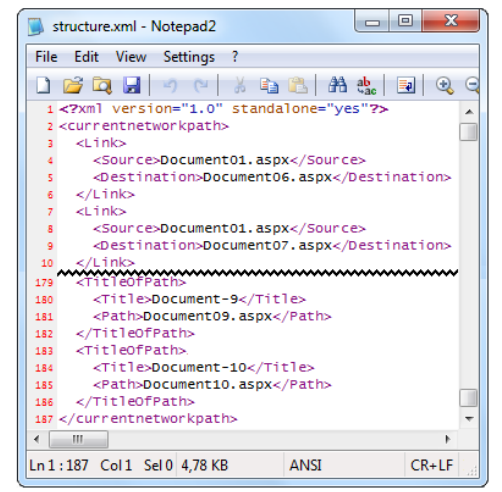

Figure 4

The parts of the file "structure.xml" obtained by an analysis. In the first (upper) part there are links between resources, and in the second (lower) part are connections between the name of the resource and its path

Picolo2D graph [13], allows changing the zoom of the whole graph, moving all the elements of the graph or each of the elements by using a mouse. If you doubleclick on any graph documents, it becomes a central element of the new graph (Figure 9), and acts as if the document has been selected in the lower section of the form in Figure 6.

\section{Directions of Further Development}

Previously mentioned software tool represents a convenient way for a website designer to take advantage of the results of the complex analysis of the previous user's behavior, and also to have a possibility to accept or reject a number of the results.

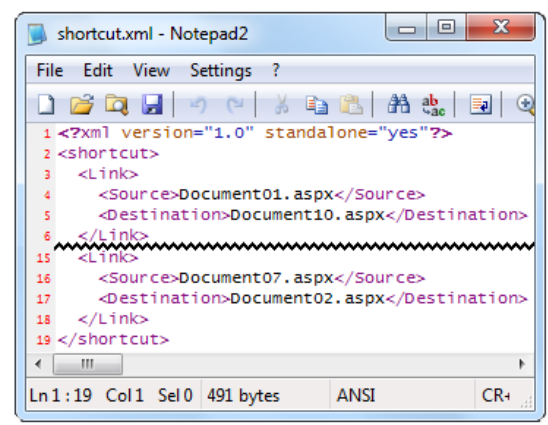

Figure 5

Recommended links between currently unrelated documents 


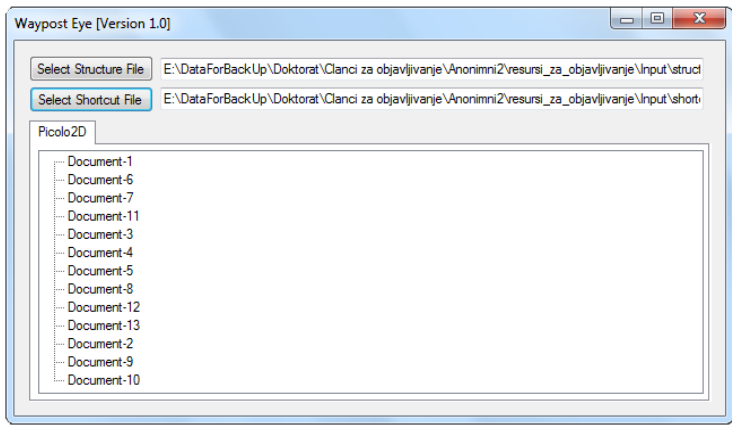

Figure 6

The main window of WaypostEye software

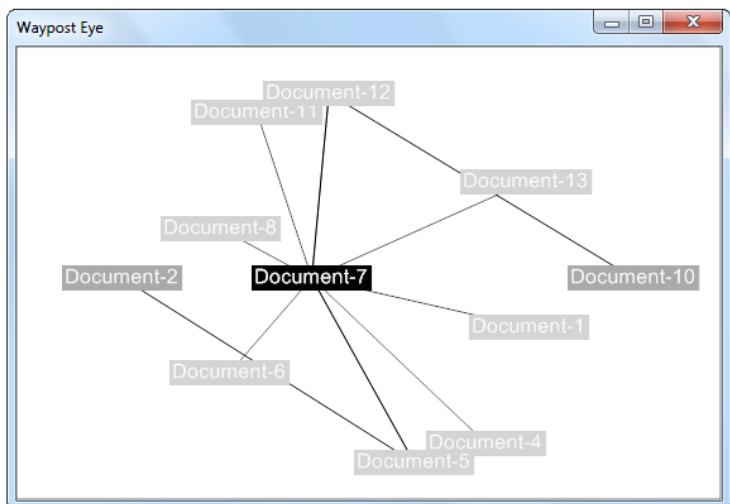

Figure 7

Graph which refers to selected document

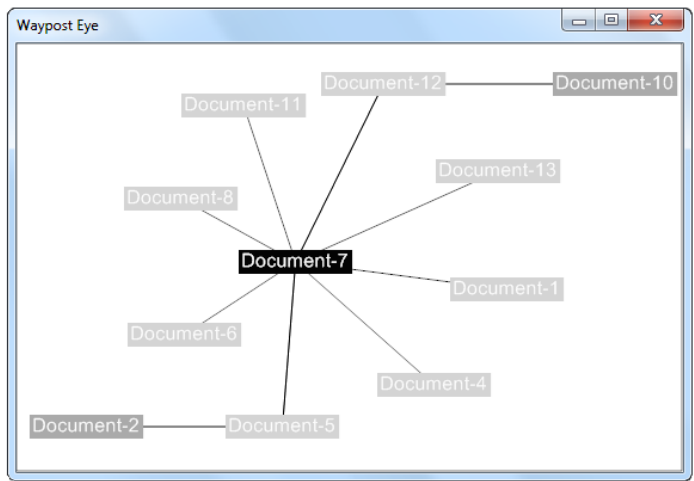

Figure 8

The graph which is related to the selected document, where by using a mouse, the resources are arranged in such a way to be more ordered 


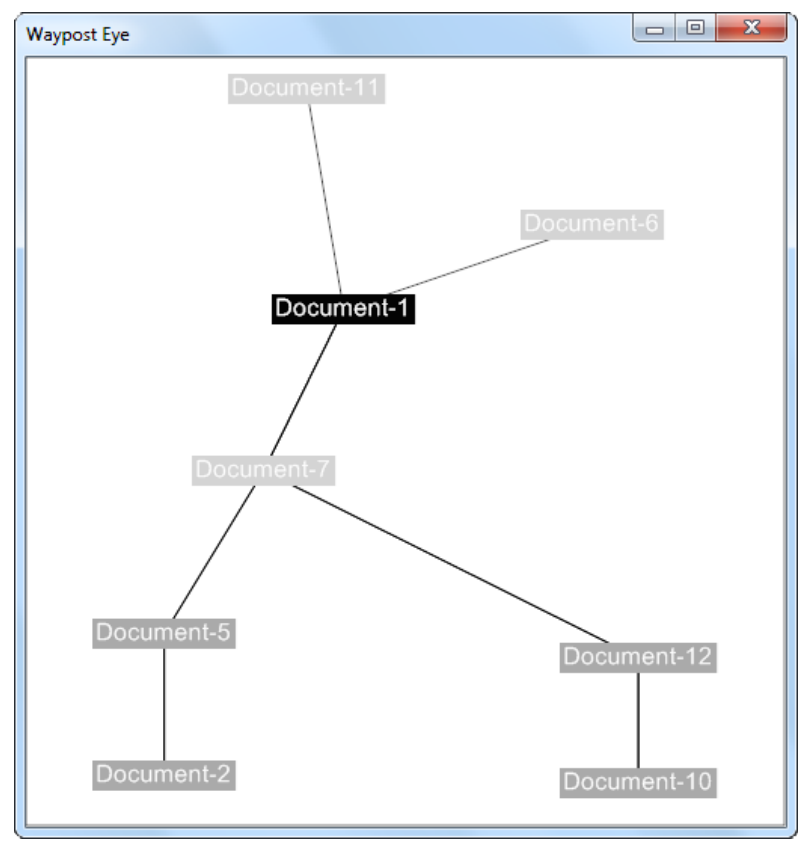

Figure 9

The graph which is related to the selected document "Document-1", where by using a mouse, the resources are arranged in such a way to be more ordered

In this way, the designer is in a position to make better business decisions in an easier way. There are several directions in which the tool could be developed in the future. The presentation of the results could be extended to various graphic and textual outputs so that the designer could choose the way of presentation that suits him best. If users are given a possibility to leave their comments during the navigation, these comments could appear in the graphs, giving the designer a possibility to read the users' comments and to take some suggestions on board.

\section{Conclusions}

One of the tasks in the process of designing a website is to create an efficient and intuitive navigation structure. Web site users often find it difficult to find a resource that they need, or the path to the resource requiring a long series of visits to various other web sites. Adaptive web sites create the possibility of the dynamic addition of links between the resources that are not connected and when there is a justification for these connections. The software, such as the one described in [5], enables us to obtain the connection proposals which should be established between resources based on the user's behavior previously recorded in log files. Such connections are not often obvious, and a serious analysis is required in order to obtain them. Current navigational structure is relatively easily obtained by HTML code analysis, which is performed by custom software. 
WaypostEye is a software tool that uses two types of input from the website:

- Arranged pairs of connections for the current navigational structure, with the connection between the resource name and the path is recorded.

- Arranged pairs of connections which should be established between formerly unrelated documents.

This software tool provides, at its output, the resource nodes that are directly linked to the selected document, as well as the documents (including the interdocuments of shortest path by Dijkstra algorithm, described in [3]), to which the selected document should be directly linked. In this version, the tool offers only one possible solution, based on the Piccolo2D [13] solution, but in the future it would be possible to easily add a variety of outputs, either graphical or textual, based on the output data obtained by this tool. The usage of this tool gives adaptive web site designers the possibility to consider the proposals for the new connections which are offered to them. A quick and straightforward business decision can be also made based on this graphical output.

\section{References}

[1] Bathumalai G.: Self Adapting Websites: Mining User Access Logs, The Robert Gordon University, Jun, 2008

[2] Dániel B., Korondi P., Sziebig G., Thomessen T.: Evaluation of Flexible Graphical User Interface for Intuitive Human Robot Interactions, Acta Polytechnica Hungarica, Vol. 11, No. 1, 2014, pp. 135-151

[3] Dijkstra E. W.: A Note on Two Problems in Connexion with Graphs, Numerische Mathematik, Vol. 1, No. 1, 1959, pp. 269-271

[4] Eremić Ž.: History-enriched Digital Objects as a Factor of Improvement of Adaptive Educational Web Site Navigation, Journal of Information Technology and Applications, Banja Luka, 2012, pp. 39-43

[5] Eremić Ž.: Navigation Improvement of Adaptive Web Sites by Using Log Files, PhD thesis, Technical faculty "Mihajlo Pupin", University of Novi Sad, Zrenjanin, 2012

[6] Eremić Ž., Radosav D.: Adaptive Web Sites in the Function of Information Access Improvement in Education, Information technology and development of education - ITRO 2011, Zrenjanin, pp. 343-347, 2011

[7] Eremić Ž., Radosav D.: Collaborative User Support as a Contribution to Navigation Improvement of Adaptive Websites for Distance Learning, Information Technology and Development of Education - ITRO 2012, Zrenjanin, 2012, pp. 265-269

[8] Eremić Ž., Radosav D., Markoski B.: Mining User Access Logs to Optimize Navigational Structure of Adaptive Web Sites, Proceedings of the CINTI 2010: $11^{\text {th }}$ IEEE International Symposium on Computational Intelligence and Informatics, Budapest, 2010, pp. 271-275 
[9] Hill W., Hollan, J.: History-enriched Digital Objects, Proceedings of Computers, Freedom: and Privacy (CFP'93), 1993, pp. 139-145

[10] Lamping J., Rao R., Pirolli P.: A Focus+Context Technique Based on Hyperbolic Geometry for Visualizing Large Hierarchies, Proceedings of CHI'95 Conference on Human Factors in Computing Systems, ACM Press, 1995, pp. 401-408

[11] Pamnani R., Chawan P.: Web Usage Mining: A Research Area in Web Mining, Paper presented at the International Conference on Recent Trends in Computer Engineering ISCET 2010, Punjab, India, 2010, pp. 73-77

[12] Perkowitz M., Etzioni O.: Adaptive Sites: Automatically Learning from User Access Patterns, In Proc. $6^{\text {th }}$ Int. World Wide Web Conf, Santa Clara, California, April 1997

[13] Piccolo2D, http://www.piccolo2d.org (Accessed on February $18^{\text {th }}$ 2013)

[14] Wexelblat A., Maes, P.: Footprints: History-Rich Tools for Information Foraging, Proceedings of the SIGCHI conference on Human factors in computing systems: the CHI is the limit, 1999, pp. 270-277 